This item was submitted to Loughborough's Research Repository by the author.

Items in Figshare are protected by copyright, with all rights reserved, unless otherwise indicated.

\title{
Gap and subgap tunnelling in cuprates
}

PLEASE CITE THE PUBLISHED VERSION

\section{LICENCE}

CC BY-NC-ND 4.0

\section{REPOSITORY RECORD}

Alexandrov, A.S., and A.F. Andreev. 2019. "Gap and Subgap Tunnelling in Cuprates". figshare. https://hdl.handle.net/2134/1141. 


\title{
Gap and subgap tunnelling in cuprates
}

\author{
A.S. Alexandrov* and A.F. Andreev** \\ * Department of Physics, Loughborough University, Loughborough LE11 3TU, United Kingdom, **P.L. Kapitza Institute for \\ Physical Problems, Kosygin st.2, 117973- Moscow, GSP-1, Russia
}

We describe strongly attractive carriers in cuprates in the framework of a simple quasi-one dimensional Hamiltonian with a local attraction. In contrast with the conventional BCS theory there are two energy scales, a temperature independent incoherent gap $\Delta_{p}$ and a temperature dependent coherent gap $\Delta_{c}(T)$ combining into one temperature dependent global gap $\Delta=\left(\Delta_{p}^{2}+\Delta_{c}^{2}\right)^{1 / 2}$. The temperature dependence of the gap and single particle (Giaver) tunnelling spectra in cuprates are quantitatively described. A framework for understanding of two distinct energy scales observed in Giaver tunnelling and electron-hole reflection experiments is provided.

PACS numbers:74.20.-z,74.65.+n,74.60.Mj

There is convincing experimental evidence that the pairing of carriers takes place well above $\mathrm{T}_{c}$ in underdoped cuprates (for a review see Ref. [i]). If carriers are paired their magnetic moments compensate each other so one could expect that the normal state uniform magnetization should fall with decreasing temperature because more and more holes are bound into singlet pairs. This unexpected drop of the normal state magnetic susceptibility was experimentally observed [2, 3] and explained in the framework of the bipolaron theory of cuprates [4, 3]. There is also a gap in the tunnelling and photoemission, which is almost temperature independent below $\mathrm{T}_{c}$ [5] and exists well above $\mathrm{T}_{c}[6]$ [], so that some segments of a 'large Fermi surface' are actually missing [9, 10]. Kinetic [11] and thermodynamic [12] data suggest that the gap opens in both charge and spin channels and exists at any relevant temperature in a wide range of doping. A plausible explanation is that the normal gap is half of the bipolaron binding energy [1], although alternative explanations have also been proposed. The temperature and doping dependence of the gap still remains a subject of controversy. Moreover, reflection experiments, in which an incoming electron from the normal side of a normal/superconducting contact is reflected as a hole along the same trajectory [13], revealed a much smaller gap edge than the bias at the tunnelling conductance maxima in a few underdoped cuprates 14. Recent intrinsic tunnelling measurements on a series of Bi '2212' single crystals 15] showed distinctly different behaviour of the superconducting and normal state gaps with the magnetic field. Such coexistance of two distinct gaps in cuprates is not well understood [16, 15].

In this letter we propose a model, which describes the temperature dependence of the gap, tunnelling spectra and electron-hole reflection in cuprates. The assumption is that the attraction potential in cuprates is large compared with the Fermi energy. The main point of our letter is independent of the microscopic nature of the attraction. Real-space pairs might be lattice and/or spin bipolarons [1], or any other preformed pairs.

We start with a generic one-dimensional Hamiltonian including the kinetic energy of carriers in the effective mass $(m)$ approximation and a local attraction potential, $V\left(x-x^{\prime}\right)=-U \delta\left(x-x^{\prime}\right)$ as

$$
\begin{aligned}
H & =\sum_{s} \int d x \psi_{s}^{\dagger}(x)\left(-\frac{1}{2 m} \frac{d^{2}}{d x^{2}}-\mu\right) \psi_{s}(x) \\
& -U \int d x \psi_{\uparrow}^{\dagger}(x) \psi_{\downarrow}^{\dagger}(x) \psi_{\downarrow}(x) \psi_{\uparrow}(x),
\end{aligned}
$$

where $s=\uparrow, \downarrow$ is the spin $\left(\hbar=k_{B}=1\right)$. The first band to be doped in cuprates is the oxygen band inside the Hubbard gap as established in polarised photoemission [17, 18]. This band is almost one dimensional as discussed in Ref. [19], so that our (quasi) one-dimensional approximation is a realistic starting point.

Solving a two-particle problem with the $\delta$-function potential one obtains a bound state with the binding energy

$$
2 \Delta_{p}=\frac{1}{4} m U^{2}
$$

and with the radius of the bound state $r=2 / m U$. We assume that this radius is less than the inter-carrier distance in cuprates, which puts a constraint on the doping level, $E_{F}<2 \Delta_{p}$, where $E_{F}$ is the free-carrier Fermi energy. Then real-space pairs are formed. If threedimensional corrections to the energy spectrum of pairs are taken into account (see, for example, Ref. [20]) the ground state of the system is the Bose-Einstein condensate. The chemical potential is pinned below the band edge by about $\Delta_{p}$ both in the superconducting and normal state [1], so that the normal state single-particle gap is $\Delta_{p}$. The binding energy $2 \Delta_{p}$ might change due to the same corrections. However, this change does not affect our further results as soon as they are expressed in terms of $\Delta_{p}$ rather than $U$.

Now we take into account that in the superconducting state $\left(T<T_{c}\right)$ the single-particle excitations interact with the condensate via the same potential $U$. Applying the Bogoliubov approximation 21 we reduce the Hamiltonian, Eq.(1) to a quadratic form as

$$
\begin{aligned}
H & =\sum_{s} \int d x \psi_{s}^{\dagger}(x)\left(-\frac{1}{2 m} \frac{d^{2}}{d x^{2}}-\mu\right) \psi_{s}(x) \\
& +\int d x\left[\Delta_{c} \psi_{\uparrow}^{\dagger}(x) \psi_{\downarrow}^{\dagger}(x)+H . c .\right],
\end{aligned}
$$


where the coherent pairing potential

$$
\Delta_{c}=-U\left\langle\psi_{\downarrow}(x) \psi_{\uparrow}(x)\right\rangle
$$

is proportional to the square root of the condensate density, $\Delta_{c}=$ constant $\times n_{0}(T)^{1 / 2}$. The single-particle excitation energy spectrum $E(k)$ is found using the Bogoliubov transformation as

$$
E(k)=\left[\left(k^{2} / 2 m+\Delta_{p}\right)^{2}+\Delta_{c}^{2}\right]^{1 / 2},
$$

if one assumes that the condensate density does not depend on position. This spectrum is quite different from the BCS quasiparticles because the chemical potential is negative, $\mu=-\Delta_{p}$. The single particle gap, $\Delta$, defined as the minimum of $E(k)$, is given by

$$
\Delta=\left[\Delta_{p}^{2}+\Delta_{c}^{2}\right]^{1 / 2} .
$$

It varies with temperature from $\Delta(0)=\left[\Delta_{p}^{2}+\Delta_{c}(0)^{2}\right]^{1 / 2}$ at zero temperature down to the temperature independent $\Delta_{p}$ above $T_{c}$. The condensate density depends on temperature as $1-\left(T / T_{c}\right)^{d / 2}$ in the ideal three $(d=3)$ and (quasi) two-dimensional $(d=2)$ Bose-gas. In the three-dimensional charged Bose-gas it has an exponential temperature dependence at low temperatures due to a plasma gap in the Bogoliubov collective excitation spectrum [22], which might be highly anisotropic in cuprates [1]. Near $\mathrm{T}_{c}$ one can expect a power law dependence, $n_{0}(T) \propto 1-\left(T / T_{c}\right)^{n}$ with $n>d / 2$ because the condensate plasmon [22] depends on temperature. The theoretical temperature dependence, Eq.(6) describes well the pioneering experimental observation of the anomalous gap in $\mathrm{YBa}_{2} \mathrm{Cu}_{3} \mathrm{O}_{7-\delta}$ in the electron-energy-loss spectra by Demuth et al [23], Fig.1, with $\Delta_{c}(T)^{2}=\Delta_{c}(0)^{2} \times\left[1-\left(T / T_{c}\right)^{n}\right]$ below $T_{c}$ and zero above $T_{c}$, and $n=4$.

The normal metal-superconductor (SIN) tunnelling conductance via a dielectric contact, $d I / d V$ is proportional to the density of states, $\rho(E)$ of the spectrum Eq.(5). Taking also into account the scattering of singleparticle excitations by a random potential, thermal lattice and spin fluctuations one finds at $T=0$ 19

$d I / d V=$ constant $\times\left[\rho\left(\frac{2 e V-2 \Delta}{\epsilon_{0}}\right)+A \rho\left(\frac{-2 e V-2 \Delta}{\epsilon_{0}}\right)\right]$,

with

$\rho(\xi)=\frac{4}{\pi^{2}} \times \frac{A i(-2 \xi) A i^{\prime}(-2 \xi)+B i(-2 \xi) B i^{\prime}(-2 \xi)}{\left[A i(-2 \xi)^{2}+B i(-2 \xi)^{2}\right]^{2}}$,

$A$ is the asymmetry coefficient [19], $A i(x), B i(x)$ the Airy functions, and $\epsilon_{0}$ is the scattering rate. We compare the conductance, Eq.(7) with one of the best STM spectra measured in $\mathrm{Ni}$-substituted $\mathrm{Bi}_{2} \mathrm{Sr}_{2} \mathrm{CaCu}_{2} \mathrm{O}_{8+x}$ single crystals by Hancottee et al [5], Fig.2. This experiment showed anomalously large $2 \Delta / T_{c}>12$ with the temperature dependence of the gap similar to that in Fig.1. The theoretical conductance, Eq.(7) describes well the anomalous $g a p / T_{c}$ ratio, injection/emission assymmetry, zero-bias conductance at zero temperature, and the spectral shape inside and outside the gap region. There is no doubt that the gap, Fig.2 is s-like, which is compatible with the phase-sensitive experiments [24] in the framework of the bipolaron theory [19]. Within the theory the single-particle gap might be almost $k$ independent while the symmetry of the Bose-Einstein condensate wave-function (i.e. of the order parameter) is $d-$ wave.

Finally, we propose a simple theory of the tunnelling into bosonic (bipolaronic) superconductor in the metallic (no-barrier) regime. As in the canonical BCS approach applied to the normal metal-superconductor tunnelling by Blonder, Tinkham and Klapwijk [25] and to the normal-superconductor boundary in the intermediate type I state by one of us [13], the incoming electron produces only outgoing particles in the superconductor $(x>l)$, allowing for a reflected electron and (Andreev) hole in the normal metal $(x<0)$. There is also a buffer layer of the thickness $l$ at the normal metalsuperconductor boundary $(x=0)$, where the chemical potential with respect to the bottom of the conduction band changes gradually from a positive large value $\mu$ in the metal to a negative value $-\Delta_{p}$ in the bosonic superconductor. We approximate this buffer layer by a layer with a constant chemical potential $\mu_{b}\left(-\Delta_{p}<\mu_{b}<\mu\right)$ and with the same strength of the pairing potential $\Delta_{c}$ as in the bulk superconductor. The Bogoliubov-de Gennes equations may be written as usual [25], with the only difference that the chemical potential with respect to the bottom of the band is a function of the coordinate $x$,

$$
\left.\begin{array}{rr}
-(1 / 2 m) d^{2} / d x^{2}-\mu(x) & \Delta_{c} \\
\Delta_{c} & (1 / 2 m) d^{2} / d x^{2}+\mu(x)
\end{array}\right) \psi(x)
$$

Thus the two-componet wave function in the normal metal is given by

$\psi_{n}(x<0)=\left(\begin{array}{l}1 \\ 0\end{array}\right) e^{i q^{+} x}+b\left(\begin{array}{l}1 \\ 0\end{array}\right) e^{-i q^{+} x}+a\left(\begin{array}{l}0 \\ 1\end{array}\right) e^{-i q^{-} x}$,

while in the buffer layer it has the form

$$
\begin{aligned}
\psi_{b}(0<x<l) & =\alpha\left(\begin{array}{c}
1 \\
\frac{\Delta_{c}}{E+\xi}
\end{array}\right) e^{i p^{+} x}+\beta\left(\begin{array}{c}
1 \\
\frac{\Delta_{c}}{E-\xi}
\end{array}\right) e^{-i p^{-} x} \\
& +\gamma\left(\begin{array}{c}
1 \\
\frac{\Delta_{c}}{E+\xi}
\end{array}\right) e^{-i p^{+} x}+\delta\left(\begin{array}{c}
1 \\
\frac{\Delta_{c}}{E-\xi}
\end{array}\right) e^{i p^{-} x},
\end{aligned}
$$

where the momenta associated with the energy $E$ are

$$
q^{ \pm}=[2 m(\mu \pm E)]^{1 / 2}
$$

and

$$
p^{ \pm}=\left[2 m\left(\mu_{b} \pm \xi\right)\right]^{1 / 2}
$$


with $\xi=\left(E^{2}-\Delta_{c}^{2}\right)^{1 / 2}$. The well-behaved solution in the superconductor with negative chemical potential is given by

$$
\psi_{s}(x>l)=c\left(\begin{array}{c}
1 \\
\frac{\Delta_{c}}{E+\xi}
\end{array}\right) e^{i k^{+} x}+d\left(\begin{array}{c}
1 \\
\frac{\Delta_{c}}{E-\xi}
\end{array}\right) e^{i k^{-} x}
$$

where the momenta associated with the energy $E$ are

$$
k^{ \pm}=\left[2 m\left(-\Delta_{p} \pm \xi\right)\right]^{1 / 2} .
$$

The coefficients $a, b, c, d, \alpha, \beta, \gamma, \delta$ are determined from the boundary conditions, which are continuity of $\psi(x)$ and its derivatives at $x=0$ and $x=l$. Applying the boundary conditions, and carrying out an algebraic reduction, we find

$$
\begin{gathered}
\quad a=2 \Delta_{c} q^{+}\left(p^{+} f^{-} g^{+}-p^{-} f^{+} g^{-}\right) / D, \\
b=-1+2 q^{+}\left[(E+\xi) f^{+}\left(q^{-} f^{-}-p^{-} g^{-}\right)\right. \\
\left.-(E-\xi) f^{-}\left(q^{-} f^{+}-p^{+} g^{+}\right)\right] / D,
\end{gathered}
$$

with

$$
\begin{aligned}
D & =(E+\xi)\left(q^{+} f^{+}+p^{+} g^{+}\right)\left(q^{-} f^{-}-p^{-} g^{-}\right) \\
& -(E-\xi)\left(q^{+} f^{-}+p^{-} g^{-}\right)\left(q^{-} f^{+}-p^{+} g^{+}\right),
\end{aligned}
$$

and $f^{ \pm}=p^{ \pm} \cos \left(p^{ \pm} l\right)-i k^{ \pm} \sin \left(p^{ \pm} l\right), g^{ \pm}=k^{ \pm} \cos \left(p^{ \pm} l\right)-$ $i p^{ \pm} \sin \left(p^{ \pm} l\right)$.

The transmisson coefficient for electrical current, $1+$ $|a|^{2}-|b|^{2}$ is shown in Fig.3 for different values of $l$ when the coherent gap $\Delta_{c}$ is smaller than the pair-breaking gap $\Delta_{p}$, and in Fig.4 for the opposite case, $\Delta_{p}<\Delta_{c}$. In the first case, Fig.3, we find two distinct energy scales, one is $\Delta_{c}$ in the subgap region due to electron-hole reflection and the other one is $\Delta$, which is the single-particle band edge. On the other hand, there is only one gap $\Delta_{c}$, which can be seen in the second case, Fig.4. We notice that the transmission has no subgap structure if the buffer layer is absent $(l=0)$ in both cases. In the extreme case of a wide buffer layer, $l>>\left(2 m \Delta_{p}\right)^{-1 / 2}$, Fig.3, or $l>>\left(2 m \Delta_{c}\right)^{-1 / 2}$, Fig.4, there are some oscillations of the transmission due to the bound states inside the buffer layer. It was shown in Ref. [4] that the pairbreaking gap $\Delta_{p}$ is inverse proportional to the doping level. On the other hand, the coherent gap $\Delta_{c}$ scales with the condensate density, and therefore with the critical temperature, determined as the Bose-Einstein condensation temperature of strongly anisotropic 3D bosons [20]. Therefore we expect that $\Delta_{p}>>\Delta_{c}$ in the underdoped cuprates, Fig.3, while $\Delta_{p} \leq \Delta_{c}$ in the optimally doped cuprates, Fig.4. Thus the model accounts for the two different gaps experimentally observed in Giaver tunnelling and electron-hole reflection in the underdoped cuprates and for a single gap in the optimally doped samples [16]. The transmission, Fig.3 and Fig.4, is entirely due to the coherent tunnelling into the condensate and (or) into the single-particle band of the bosonic superconductor. There is also an incoherent transmission into localised single-particle impurity states and into incoherent ('supracondensate') bound pair states, which might explain a significant featureless background in the subgap region [14].

In conclusion, we have proposed a simple general model, which provides an explanation of the temperature dependence of the gap and of the single-particle tunnelling spectra in cuprates. The main assumption is that the attractive potential is large compared with the Fermi energy, so that the ground state is the Bose-Einstein condensate of tightly bound pairs. We have developed a theory of tunnelling in the metallic regime with no barrier and found two different energy scales in the transmission as observed experimentally.

We acknowledge support of this work by the Leverhulme Trust (London), grant VP/261.

[1] A.S. Alexandrov and N.F. Mott, Rep. Prog. Phys. 57 1197 (1994).

[2] D.C. Johnston, Phys. Rev. Lett 62, 957 (1989).

[3] K.A. Müller et al, J.Phys.: Condens. Matter 10, L291 (1998).

[4] A.S. Alexandrov, V.V. Kabanov and N.F. Mott, Phys. Rev. Lett. 77, 4796 (1996)

[5] H. Hancotte et al, Phys. Rev. B55, R3410 (1997).

[6] Ch. Renner et al, Phys. Rev. Lett. 80, 149 (1998).

[7] A. Mourachkine, Europhys. Lett. 49, 86 (2000).

[8] Z.-X. Shen and J.R. Schrieffer, Phys. Rev. Lett. 78, 1771 (1997) and references therein.

[9] N.L. Saini et al, Phys. Rev. Lett. 79, 3467 (1997).

[10] N.L. Saini et al, Phys. Rev. Lett. 82, 2619 (1999)

[11] B. Batlogg et al, Physica C (Amsterdam) 135-140, 130 (1994);

[12] J.W. Loram et al, Physica C (Amsterdam), 235, 134 (1994).

[13] A.F. Andreev, Zh. Eksp. Teor. Fiz. 46, 1823 (1964) [Sov. Phys. JETP 19, 1228 (1964)].

[14] Y. Yagil et al, Physica C (Amsterdam) 250, 59 (1995).

[15] P. Müller et al, unpublished.

[16] G. Deutscher, Nature 397, 410 (1999).

[17] M. C. Schabel et al, Phys. Rev. B 57, 6090 (1998).

[18] A.S. Alexandrov and C.J. Dent, Phys. Rev. 60, 15414 (1999).

[19] A.S. Alexandrov, Physica C (Amsterdam) 305, 46 (1998).

[20] A.S. Alexandrov and V.V. Kabanov, Phys. Rev. B 59, 13628 (1999).

[21] N. Bogoliubov, J.Phys. USSR 11, 23-32(1947).

[22] L.L. Foldy, Phys.Rev. 124, 649(1961).

[23] J.E. Demuth et al, Phys. Rev. Lett. 64, 603 (1990)

[24] D.A. Wollman et al, Phys. Rev. Lett. 71, 2134 (1993); C.C. Tsuei et al, Phys. Rev. Lett. 73, 593 (1994); J.R. Kirtley et al, Nature 373, 225 (1995); C.C. Tsuei et al, Science 272, 329 (1996). 
[25] G.E. Blonder, M. Tinkham, and T.M. Klapwijk, Phys. Rev. B25, 4515 (1982).

\section{Figure Captures}

Fig.1. Temperature dependence of the gap, Eq.(6) (line) compared with the experiment [23](dots) for $\Delta_{p}=$ $0.7 \Delta(0)$.

Fig.2. Theoretical tunnelling conductance, Eq.(7) (line) compared with STM conductance in Ni-doped $\mathrm{Bi}_{2} \mathrm{Sr}_{2} \mathrm{CaCu}_{2} \mathrm{O}_{8+x}$ [5]) (dots) for $2 \Delta=90 \mathrm{meV}, A=$ $1.05, \epsilon_{0}=40 \mathrm{meV}$.

Fig.3. Transmission versus voltage (measured in units of $\left.\Delta_{p} / e\right)$ for $\Delta_{c}=0.2 \Delta_{p}, \mu=10 \Delta_{p}, \mu_{b}=2 \Delta_{p}$ and $l=0$ (thick line), $l=1$ (thick dashed line), $l=4$ (thin line), and $l=8$ (thin dashed line) (in units of $1 /\left(2 m \Delta_{p}\right)^{1 / 2}$ ).

Fig.4. Transmission versus voltage (measured in units of $\left.\Delta_{c} / e\right)$ for $\Delta_{p}=0.2 \Delta_{c}, \mu=10 \Delta_{c}, \mu_{b}=2 \Delta_{c}$ and $l=0$ (thick line), $l=1$ (thick dashed line), $l=4$ (thin line), and $l=8$ (thin dashed line) (in units of $1 /\left(2 m \Delta_{c}\right)^{1 / 2}$ ). 
arXiv:cond-mat/0005315 v3 25 May 2000

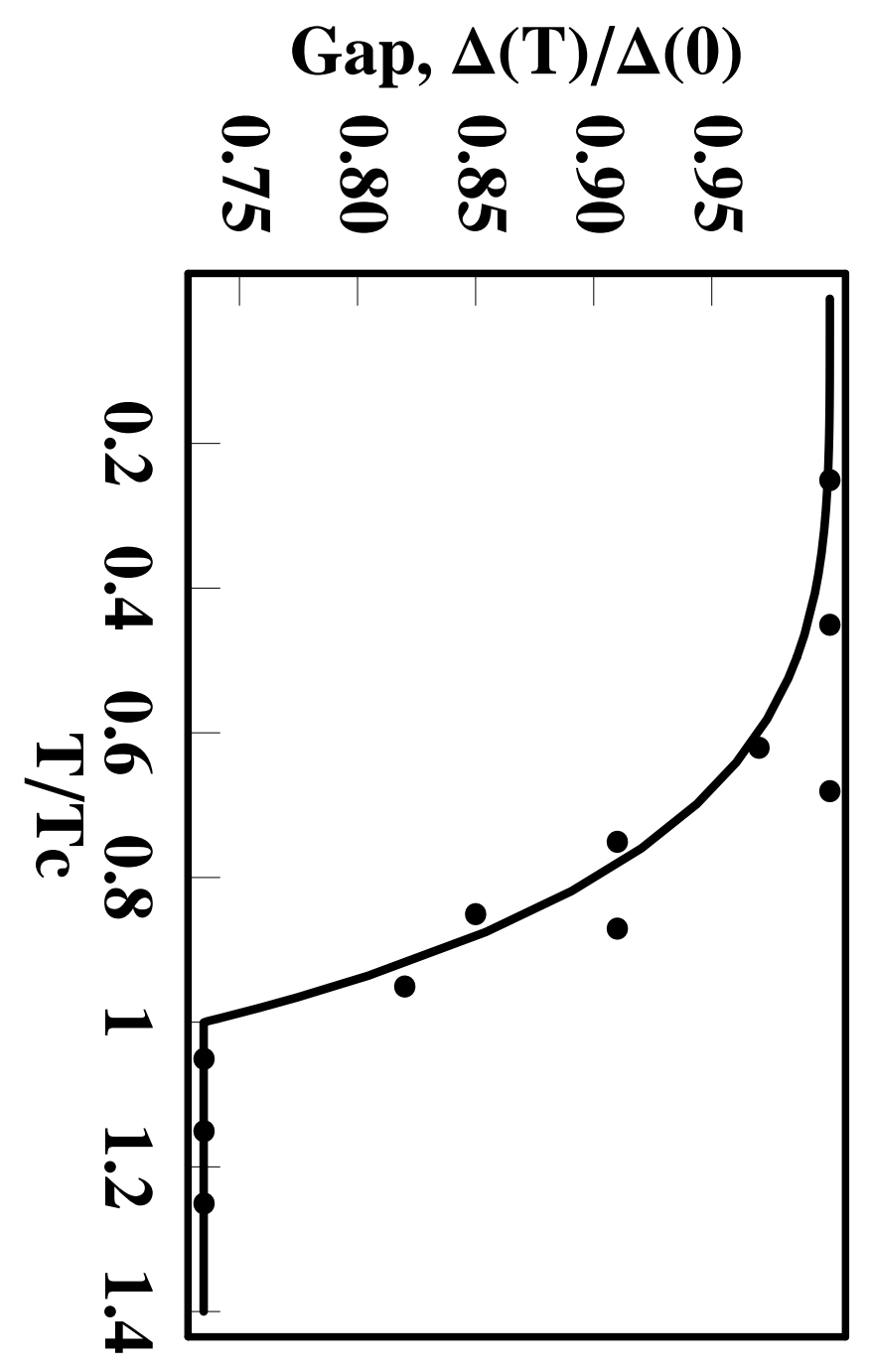




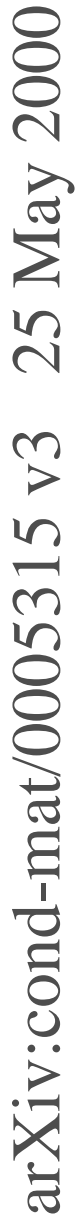

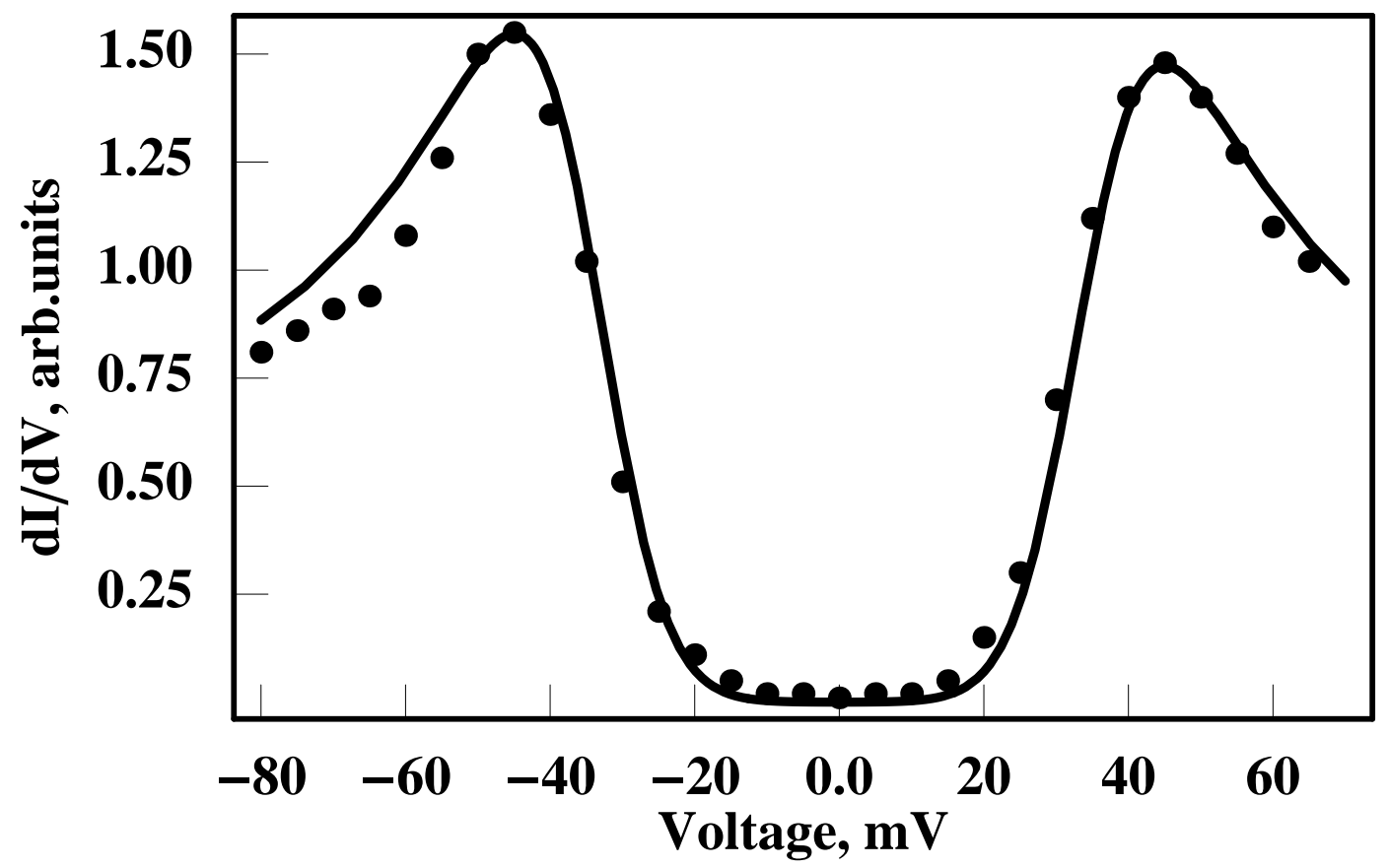


arXiv:cond-mat/0005315 v3 25 May 2000

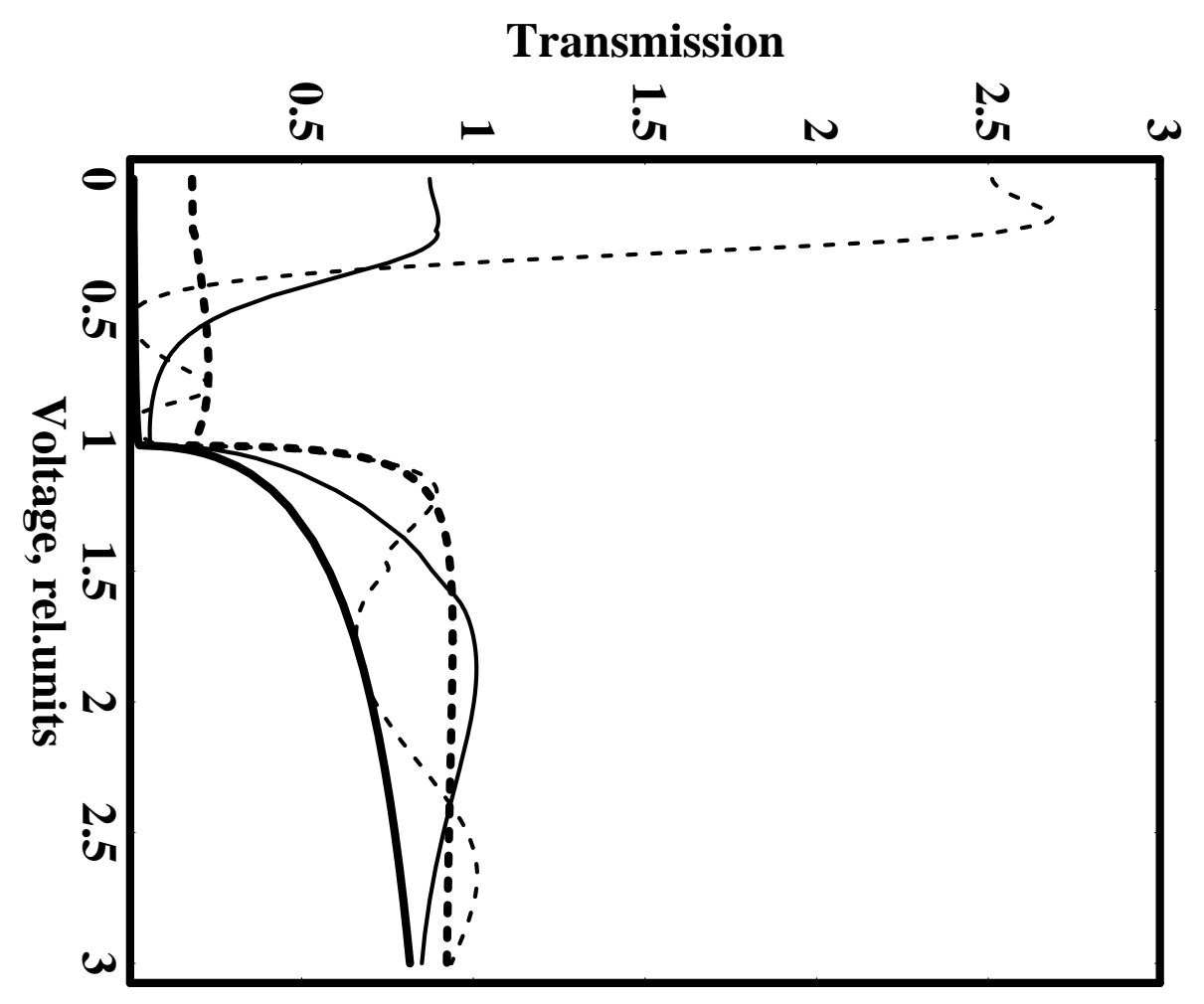


arXiv:cond-mat/0005315 v3 25 May 2000

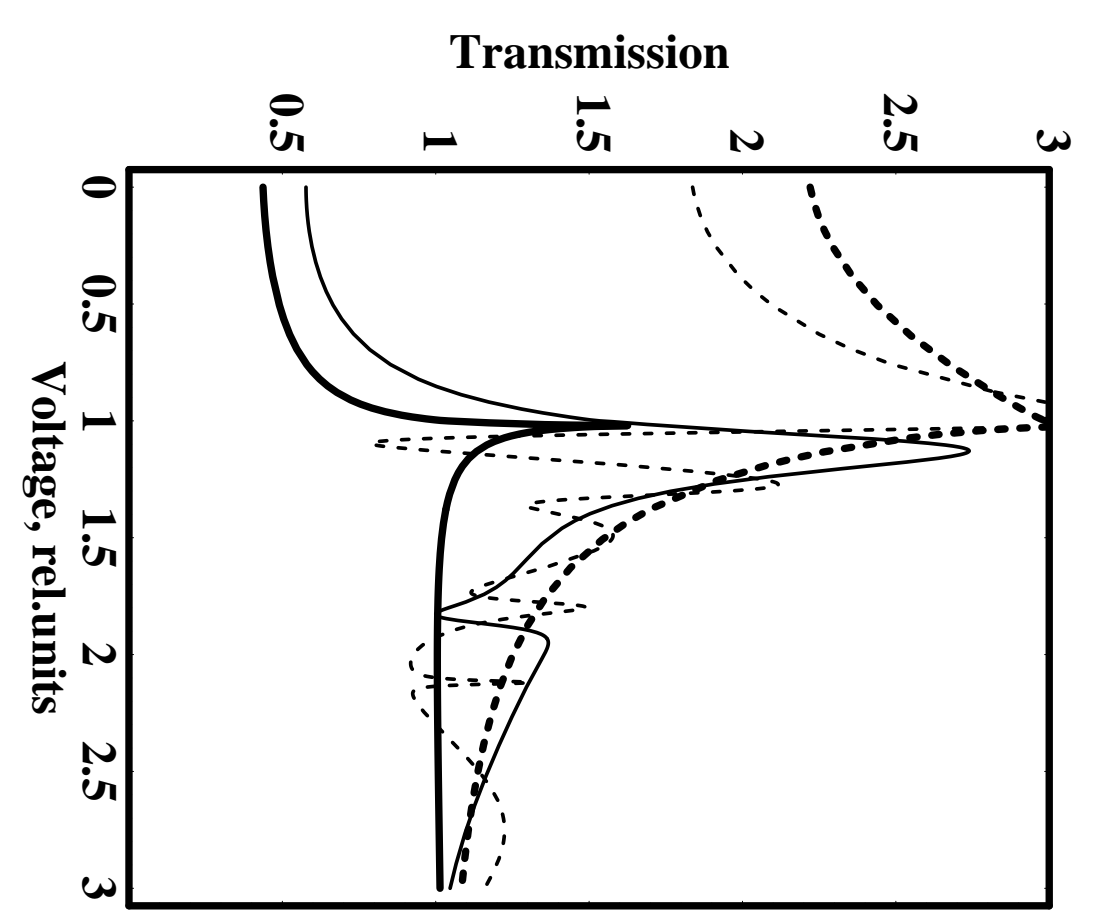

